To the Editors:

\title{
The role of flexible sigmoidoscopy in the evaluation of isolated rectal bleeding
}

\author{
U Jayarajah ${ }^{1}$, VC de Silva², DN Samarasekera ${ }^{3}$
}

Ceylon Medical Journal 2017; 62: 250-52

DOI: http://doi.org/10.4038/cmj.v62i4.8580

Flexible sigmoidoscopy is done routinely for isolated rectal bleeding in some surgical units, even when a cause is identified on clinical examination and proctoscopy, irrespective of the age of the patient and the nature of the clinical symptoms and signs. This is primarily due to the fear of missing a sinister cause such as carcinoma of the colon or rectum [1]. However, routine flexible sigmoidoscopy in all patients may impose a considerable burden on the cost of healthcare, particularly in a developing country, where the resources are limited [1]. Therefore, this study was aimed at describing the yield of flexible sigmoidoscopy in the evaluation of isolated rectal bleeding.

A retrospective analysis of prospectively entered data was done. All patients who underwent flexible sigmoidoscopy for isolated rectal bleeding from January 2007 to October 2015 at the Professorial Surgical Unit, National Hospital of Sri Lanka were analysed. Ethical clearance was obtained from the Ethics Review Committee of National Hospital of Sri Lanka.

Those who had other additional lower gastrointestinal symptoms, palpable rectal mass and previous history of colorectal malignancies and inflammatory bowel disease were excluded. Digital rectal examination and proctoscopy was done in all patients.

Of a total of 3283 patients who underwent sigmoidoscopy, 670 patients underwent flexible sigmoidoscopy (standard $60 \mathrm{~cm}$-fibreoptic flexible sigmoidoscope, Olympus-CFP20S, Japan) for isolated rectal bleeding. If an additional lesion was identified, a colonoscopy was performed for further assessment. The patients were grouped into two categories, i.e. age 40 years or less and age more than 40 years and the yield of sigmoidoscopy was compared between the two groups.

The mean age was $46.0 \pm \mathrm{SD} 15.58$ (range: $18-94$ ) years. Of those, $257(38.4 \%)$ patients were aged 40 years or less. The majority $(n=400 ; 59.6 \%$, ) were males.
The majority had haemorrhoids as the only abnormality $(n=406 ; 60.5 \%)$. Clinical examination, proctoscopy and flexible sigmoidoscopy were normal in $154(22.98 \%)$ patients. Polyps were found in 35(5.2\%), inflammatory lesions in $51(7.6 \%)$ and diverticulae in $3(0.4 \%)$ patients. Presence of erythema, oedema and ulcers were considered as inflammatory lesions. Eleven patients $(1.6 \%)$ had less common findings such as, vascular malformations, worm infestation and mucosal prolapse. The majority $(n=559 ; 83.5 \%)$ did not have additional lesions apart from those detected at clinical examination and proctoscopy. Thus, additional findings were detected only in $16.5 \%$ of all patients.

\section{Neoplastic lesions}

Fourteen patients (2.1\%) had colorectal adenocarcinoma of which, 9 occurred as isolated lesions and 4 occurred in the background of an adenomatous polyp. Six patients had invasive adenocarcinoma. One patient was found to have carcinoma as a dual pathology in addition to haemorrhoids. One patient aged less than 40 years had a rectal malignancy as the sole cause for rectal bleeding.

Polyps were detected in $35(5.2 \%)$ patients. (isolated $=28$, dual finding $=7)$. The majority $(n=13$; $37.14 \%$ ) were adenomatous polyps (tubular adenoma $=9$, tubulovillous adenoma $=3$ and villous adenoma $=1)$. Four polyps showed histological evidence of malignancy. However, all polyps identified as dual pathology were histologically benign (hyperplastic polyp $=4$, tubular adenoma $=2$, unremarkable $=1$ ).

\section{Inflammatory lesions}

Histological assessment of 28 (54.9\%) patients with inflammatory lesions were unremarkable while

1Department of Surgery, Faculty of Medicine, University of Colombo, 2Chair Professor of Pathology, Faculty of Medicine, University of Colombo, 3Chair Professor of Surgery, Faculty of Medicine, University of Colombo

Correspondence: DNS, e-mail: <samarasekera58@yahoo.co.uk>. Received 21 August 2017 and revised version accepted 14 September 2017 
Table 1. Yield of flexible sigmoidoscopy according to age and sex

\begin{tabular}{|c|c|c|c|c|c|c|}
\hline & \multicolumn{3}{|c|}{40 year or less } & \multicolumn{3}{|c|}{ More than 40 years } \\
\hline & Male N (\%) & $\begin{array}{l}\text { Female N } \\
(\%)\end{array}$ & $\begin{array}{l}\text { Total N } \\
\text { (\%) }\end{array}$ & Male N (\%) & $\begin{array}{l}\text { Female } N \\
(\%)\end{array}$ & $\begin{array}{l}\text { Total N } \\
\text { (\%) }\end{array}$ \\
\hline \multicolumn{7}{|l|}{ Isolated findings } \\
\hline Haemorrhoids & $\begin{array}{l}100(63.7 \\
\%)\end{array}$ & $52(52.0 \%)$ & $152(59.1 \%)$ & $158(65.0 \%)$ & $101(59.4 \%)$ & $259(62.7 \%)$ \\
\hline Malignancy & $0(0.0 \%)$ & $1(1.0 \%)$ & $1(0.4 \%)$ & $5(2.1 \%)$ & $7(4.1 \%)$ & $12(2.9 \%)$ \\
\hline Normal & $40(25.5 \%)$ & $30(30.0 \%)$ & $70(27.2 \%)$ & $42(17.3 \%)$ & $42(24.7 \%)$ & $84(20.3 \%)$ \\
\hline Benign adenomatous polyp & $0(0.0 \%)$ & $1(1.0 \%)$ & $1(0.4 \%)$ & $6(2.5 \%)$ & $4(2.4 \%)$ & $10(2.4 \%)$ \\
\hline Inflammatory & $9(5.7 \%)$ & $10(10.0 \%)$ & $19(7.4 \%)$ & $10(4.1 \%)$ & $6(3.5 \%)$ & $16(3.9 \%)$ \\
\hline Diverticula & $0(0.0 \%)$ & $0(0.0 \%)$ & $0(0.0 \%)$ & $1(0.4 \%)$ & $2(1.2 \%)$ & $3(0.7 \%)$ \\
\hline Other benign findings & $5(3.2 \%)$ & $5(5.0 \%)$ & $10(3.9 \%)$ & $10(4.1 \%)$ & $4(2.4 \%)$ & $14(3.4 \%)$ \\
\hline \multicolumn{7}{|l|}{ Dual pathology } \\
\hline Malignancy + haemorroids & $0(0.0 \%)$ & $0(0.0 \%)$ & $0(0.0 \%)$ & $0(0.0 \%)$ & $1(0.6 \%)$ & $1(0.2 \%)$ \\
\hline adenomatous polyp + & $0(0.0 \%)$ & $0(0.0 \%)$ & $0(0.0 \%)$ & $2(0.8 \%)$ & $0(0.0 \%)$ & $2(0.5 \%)$ \\
\hline
\end{tabular}

inflammatory bowel disease was detected in 9 patients. Of the inflammatory lesions identified as dual pathology, $2(12.5 \%)$ had inflammatory bowel disease, $1(6.25 \%)$ had collagenous colitis and the rest $(n=13,81.25 \%)$ showed no histological changes.

\section{Lesions identified as dual pathology}

Nineteen $(2.8 \%)$ patients had dual pathology and all were in addition to haemorrhoids. All dual pathologies $(n=4 ; 1.6 \%)$ detected in those aged 40 years or less were benign and were located in the rectum. Of the dual pathologies detected in those aged more than 40 years $(n=15)$, one patient had a malignancy. (Table 1)

A similar retrospective analysis of 1275 patients by Mathew et al reported that in those aged more than 45 years, $3.5 \%$ had colorectal cancers and $6.8 \%$ had adenomatous polyps [1]. Of those less than 45 years, none had a malignancy and $2.1 \%$ had adenomatous polyps [1]. A study of 337 patients aged more than 40 years who presented with frank rectal bleeding, showed that a considerable number had neoplastic lesions; i.e. $9.1 \%$ had cancers and $10.3 \%$ had polyps [2]. In a study carried out by Bhatti et al on 215 patients with rectal bleeding, $7.1 \%$ had malignancies and $17.2 \%$ had polyps [3]. In a study by Manzoor et al in 200 patients with rectal bleeding, colorectal carcinoma was seen among $8 \%$ of patients [4]. Rectal prolapse $(2.5 \%)$, polyps $(2.0 \%)$ and ulcerative colitis $(1.0 \%)$ were other causes detected. The considerably higher yield in the above studies may be due to the inclusion of patients with other concomitant gastrointestinal symptoms whereas in the present study only those with isolated rectal bleeding were included.

Routine flexible sigmoidoscopies impose a higher cost, especially in a setting with limited resources and subjects patients to discomfort and complications due to endoscopy. Although the yield of dual pathology was considerably low in those aged less than 45 years, we detected one patient with a malignancy. Although rare, missing a sinister cause in a young patient is not acceptable as it causes significant morbidity and will affect prognosis.

Rigid sigmoidoscopy is widely used as a preliminary investigation to look for colorectal pathology. It is a relatively cheap procedure that can be done in the outpatient setting on unprepared bowel and can be utilised in settings with limited resources for evaluation of rectal bleeding [5]. In a study by Nikpour et al in 402 patients with fresh rectal bleeding, all significant lesions in young patients were within the reach of rigid sigmoidoscopy [6]. However, studies have proven that flexible sigmoidoscopy has better diagnostic yield and less patient discomfort [5]. 
There is no doubt that at least a flexible sigmoidoscopy is essential for evaluation of older patients. We suggest that a flexible sigmoidoscopy should be ideally offered for evaluation of isolated fresh rectal bleeding in all young patients irrespective of the clinical assessment and proctoscopy. However, in a resource limited setting where flexible sigmoidoscopy is not accessible, a rigid sigmoidoscopy should be the minimum standard.

\section{Conflicts of interest}

Authors declare that there are no conflicts of interest.

\section{References}

1. Mathew J, Shankar P, Aldean IM. Audit on flexible sigmoidoscopy for rectal bleeding in a district general hospital: are we over-loading the resources? Postgrad Med J 2004; 80: 38-40.

2. Cheung PS, Wong SK, Boey J, Lai CK. Frank rectal bleeding: a prospective study of causes in patients over the age of 40. Postgrad Med J 1988; 64: 364-8.

3. Bhatti ABH, Quraishy MS. Flexible sigmoidoscopic findings in patients with fresh rectal bleeding. J Coll Physicians Surg Pak 2011; 21: 577-8.

4. Manzoor A, Shah S, Inam A. Etiologic spectrum of bleeding per Rectum in surgical outpatient department of a tertiary care hospital. Annals of Pakistan Institute of Medical Sciences 2011; 7: 180-5.

5. Rao VS, Ahmad N, Al-Mukhtar A, Stojkovic S, Moore PJ, Ahmad SM. Comparison of rigid vs flexible sigmoidoscopy in detection of significant anorectal lesions. Colorectal Dis 2005; 7: 61-4.

6. Nikpour S, Asgari AA. Colonoscopic evaluation of minimal rectal bleeding in average-risk patients for colorectal cancer. World J Gastroenterol 2008; 14: 6536-40. 